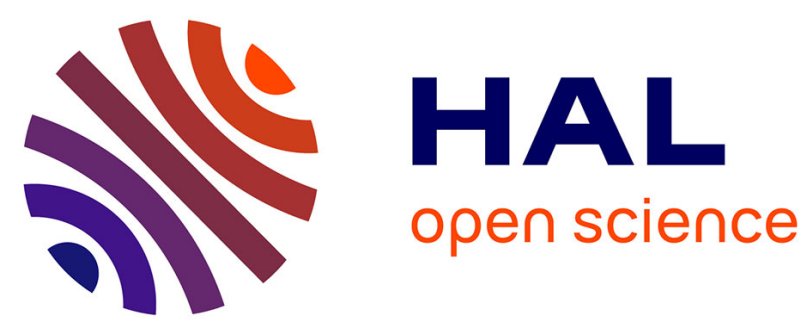

\title{
DATA HIDING OF INTRA PREDICTION INFORMATION IN CHROMA SAMPLES FOR VIDEO COMPRESSION
}

Jean-Marc Thiesse, Joël Jung, Marc Antonini

\section{- To cite this version:}

Jean-Marc Thiesse, Joël Jung, Marc Antonini. DATA HIDING OF INTRA PREDICTION INFORMATION IN CHROMA SAMPLES FOR VIDEO COMPRESSION. 2010 IEEE 17th International Conference on Image Processing (ICIP), Sep 2010, Hong Kong, Hong Kong SAR China. pp.2861. hal-00526531

\section{HAL Id: hal-00526531 \\ https://hal.science/hal-00526531}

Submitted on 14 Oct 2010

HAL is a multi-disciplinary open access archive for the deposit and dissemination of scientific research documents, whether they are published or not. The documents may come from teaching and research institutions in France or abroad, or from public or private research centers.
L'archive ouverte pluridisciplinaire HAL, est destinée au dépôt et à la diffusion de documents scientifiques de niveau recherche, publiés ou non, émanant des établissements d'enseignement et de recherche français ou étrangers, des laboratoires publics ou privés. 


\title{
DATA HIDING OF INTRA PREDICTION INFORMATION IN CHROMA SAMPLES FOR VIDEO COMPRESSION
}

\author{
J-M. Thiesse ${ }^{1}$, J. Jung ${ }^{1}$, and M. Antonini ${ }^{2}$ \\ ${ }^{1}$ Orange Labs \\ 38 rue du G. Leclerc, 92794 Issy les Moulineaux, France \\ \{jeanmarc.thiesse, joelb.jung\}@ orange-ftgroup.com \\ 2 I3S Lab. University of Nice-Sophia Antipolis/CNRS \\ 2000 route des Lucioles, 06903 Sophia Antipolis, France \\ am@i3s.unice.fr
}

\begin{abstract}
New activities have been recently launched in order to challenge the H.264/AVC standard. Several improvements of this standard are already known, however the targeted $50 \%$ bitrate saving for equivalent quality is not yet achieved.

In this context, a previous work proposes to use data hiding techniques to reduce the signaling information resulting from an improvement of Inter-coding. The main idea is to hide the indices into appropriately selected chroma and luma transform coefficients. To minimize the prediction errors, the modification is performed via a rate-distortion optimization.

In this paper, the scheme is extended for Intra-coding and 4:4:4 sequences in order to explore the limits highlighted by the previous study and tackle some remaining issues. A different rate-distortion optimization built on the Pareto theory is also proposed. Resulting improvements (1.5\% in average) for several sequences are reported and analyzed.
\end{abstract}

Index Terms - Video coding, competition index signalization, chroma sample, data hiding.

\section{INTRODUCTION}

Significant compression gain, compared to former video coding standards, has been achieved by the H.264/AVC standard [1] resulting from the joint work of the ITU-T SG16-Q6 and ISO/IEC Moving Pictures Experts Group (MPEG). This gain results from the improvement of existing tools and the inclusion of new ones. These improvements concern the motion estimation, the information coding with Context Adaptive Binary Arithmetic Coding (CABAC), and above all the addition of several Intra and Inter modes with many encoding methods which need the transmission of competition signalization indices.

Today, new video coding standard activities are launched. Both VCEG (Video Coding Expert Group) and MPEG are working collaboratively, through the JCT-VC (Joint Collaborative Team on Video Coding) and have just issued a Call for Proposals with answers given in April 2010. The goal is to reach a standard that provides a bitrate reduction of $50 \%$ at the same subjective quality, with a complexity increased by a factor 2 or 3. Several improvements are already known and gathered in the JM KTA software (Key Technical Area) [2]. Some of these improvements and others contributions add new competition indices which are more and more costly. We have proposed in a previous work [3] to deal with the reduction of this cost through a framework that allows to hide such indices in chroma and luma texture samples. In this paper, we propose to explore the limits highlighted by the previous study which has been performed in Inter-coding. The scheme is first extended for Intra-coding with an optimal selection of hidden indices and we propose a different rate-distortion optimization built on the Pareto theory. The scheme is also tested for 4:4:4 color format sequences which, as Intra blocks, allow to hide more indices in chroma sample.

The remaining of this paper is organized as follows: in Section 2, an H.264/AVC and data hiding state of the art is presented, including the major conclusions of our previous work. Section 3 gives the details of our index hiding scheme and the proposed extensions. Finally the experimental results are reported and analyzed in Section 4.

\section{STATE OF THE ART}

\subsection{H.264/AVC prediction and coding}

H.264/AVC is an hybrid video coder: Intra and Inter prediction are used together to exploit spatial and temporal redundancies. For each macroblock, several modes are in competition and need to be signaled to the decoder with other information of residual or coding type (submode, DCT size,...).

All of them are stored sequentially in the bitstream and moreover independently one from the other. In order to reduce this cost, a Most Probable Mode (MPM) is used: the mode that is the most probable is encoded with 1 bit only. Although context adaptive encoding is performed, the cost with these existing methods remains high. At medium bitrate, the competition information represents $20 \%$ of the total bitrate and this proportion increases up to $40 \%$ for lower bitrates.

In this paper, we propose to use data hiding for decreasing the rate cost of these competition indices. 


\subsection{Data Hiding}

Data hiding and video compression traditionally have contradictory goals. The first one adds imperceptual information to embed data, while the second removes redundant information to reduce video size. More precisely, data hiding deals with the ability of embedding data into a digital media with a minimum visible degradation. For each data hiding applications, a complex trade-off between three parameters is needed: data playload (amount of informations to hide), fidelity (distortion induced by the mark) and robustness (resistance to attacks).

Three kinds of data hiding approaches can be reported. The first extends still image watermarking, the second exploits the temporal dimension of video in order to increase the fidelity and the robustness [4]. The third class is based on the video compression standard. Among the latter class, most methods hides informations in the non-zero DCT coefficients of a compressed video stream. The force even watermarking [5] is one of the most widespread approach: it hides information in the parity of $\mathrm{AC}$ coefficients at the cost of a bitrate increase. An other solution is proposed in [6] by embedding each bit on the parity sum of the transform coefficients in order to choose the modification which induce the smallest degradation.

In addition to classical applications of data hiding, a few methods only propose to use data hiding for compression purposes, as in our paper. It has been formalized for still image coding in [7] where authors propose to hide the chrominance information in the wavelet domain of the luminance component before image compression. This paper and some related approaches $[8,9]$ confirm the potential great interest of data hiding methods for compression. Contrary to these approaches, our paper proposes an optimal way to introduce such technique in a video compression standard like H.264/AVC.

\subsection{Data hiding for video compression in Inter-coding}

In our previous work [3], we have proposed to hide motion competition information generated by the Motion Vector Competition (MVComp) tool [10] in transform and quantized coefficients of Luma and Chroma residuals. More precisely, hiding in the parity of the sum of the transform coefficients permits to easily balance between rate and distortion modifications. Although the paper solves initial difficulties related to this original video compression approach, the average bitrate savings remain low. Some important issues were reported and need to be further adressed, such as:

1. The amount of coefficients available for hiding.

2. The selection of the data to hide.

3. The selection of the coefficient to be modified.

In this new study, we explore these issues in order to extend the first work, and provide increased bitrate savings.

\section{PROPOSED SCHEME AND EXTENSIONS}

\subsection{Preliminary study}

As explained in the previous section, we have favored a scheme which embeds the mark directly into the compressed video stream, especially into the pixel residuals. In order to overcome the issues reported in [3], we propose in a first extension to deal whith Intra-coding as the Intra contains more non-zero transform coefficients, especially in chroma samples. Among all the Intra competition indices, the MPM index is chosen because it is the most expensive $(6 \%$ of the total Intra bitrate at medium bitrate). This is a binary index which indicates if the Intra submode to encode is equal or not to the most probable mode defined by the neighbours blocks.

Our second extension consists in applying the scheme for 4:4:4 color format sequences. This format allows to hide more coding information because there is potentially four times more Chroma blocks with non zero coefficients. Moreover, each index can be hidden in more coefficients and there is consequently less impact on the reconstructed pixels.

Finally, the issue of setting the Lagrangian parameters in the previously proposed $\mathrm{RD}$ approach is tackled using the Pareto theory.

\subsection{Scheme description}

We use the Chroma quantized and transform coefficients to hide the MPM index. This hiding is sensitive because of the specificities of CABAC and the possible prediction damages. The proposed scheme satisfies the following requirements:

1. Control of the bit-rate change: it must be lower than the index signaling, $R_{i}^{w}-R_{i}<\varsigma\left(I_{i}\right)$, where $R_{i}$ and $R_{i}^{w}$ are the original and the modified rate of the $i^{\text {th }}$ block, and $I_{i} \in\{0,1\}$ is the MPM index with $\varsigma\left(I_{i}\right)$ its cost.

2. Minimization of prediction degradation: the transform coefficients modification must be invisible and must have low impact on the prediction of the next frames.

In order to meet these requirements, we use the parity of the coefficients sum to mark the MPM index. The first requirement can be met by rejecting all the modifications which increase the original bit-rate too much. For the second requirement, Pareto based optimization is applied.

Let us note $a_{n}, n \in\{1, \ldots, N\}$ the transform and quantized coefficients before transmission. The sum of the coefficients of the $i^{\text {th }}$ block is denoted as $S_{i}=\sum_{n=1}^{N} a_{n}$.

The resulting modified $S_{i}^{w}$ is obtained as follow:

$$
S_{i}^{w}= \begin{cases}S_{i} & ;\left|S_{i}\right| \bmod 2=I_{i} \\ S_{i}+m_{i} & ;\left|S_{i}\right| \bmod 2 \neq I_{i},\end{cases}
$$

where $I_{i}$ is the index and $m_{i}$ is the coefficients shift. Blocks with DC coefficients only are not used to limit the damages. 
We propose to use a Pareto optimization to choose the best modification when the coefficients need to be modified, i.e: $I_{i} \neq\left|S_{i}\right| \bmod 2$. We consider $N$ transform coefficients $a_{n}, a_{n} \neq 0$. For each coefficient, 6 rate-distortion pairs $\left(R_{n}^{w_{j}}, D_{n}^{w_{j}}\right)$ are computed after addition of an odd value $m_{j}$ :

$$
a_{n}^{w_{j}}=a_{n}+m_{j}
$$

We choose to limit the $m_{j}$ values to $\{-5,-3,-1,1,3,5\}$ because larger ones induce too high signal modification. $R_{n}^{w_{j}}$ is the rate generated by the coding of the modified coefficients and $D_{n}^{w_{j}}$ is the related distortion. The null transform coefficients are not considered to avoid breaking the zero series. Note that each index can be hidden equivalently in $\mathrm{U}$ or $\mathrm{V}$ chroma component.

As an alternative to the classical RD-optimization used in previous study [3], we apply here a method based on the theory of the multicriteria optimization to choose the best modification. Built on the Pareto theory, it consists in three steps:

1. Computation of the rate-distortion pairs $\left(\bar{R}_{n}^{w_{j}}, \bar{D}_{n}^{w_{j}}\right)$ which correspond to a Pareto-optimal pair $\left(\bar{a}_{n}, \bar{m}_{j}\right)$. A given pair of variables, $\left(\bar{a}_{n}, \bar{m}_{j}\right)$, is Pareto-optimal if and only if it is not dominated by any other $\left(a_{n}, m_{j}\right)$, where $\left(a_{n}, m_{j}\right)$ dominates $\left(\bar{a}_{n}, \bar{m}_{j}\right)$ means:

$$
\begin{gathered}
\text { either }:\left\{\left(R_{n}^{w_{j}} \leq \bar{R}_{n}^{w_{j}}\right) \text { and }\left(D_{n}^{w_{j}}<\bar{D}_{n}^{w_{j}}\right)\right\}, \\
\text { or }:\left\{\left(R_{n}^{w_{j}}<\bar{R}_{n}^{w_{j}}\right) \text { and }\left(D_{n}^{w_{j}} \leq \bar{D}_{n}^{w_{j}}\right)\right\}
\end{gathered}
$$

Figure 1 plots an example of a Pareto frontier. The points represent the 6 possible modifications for a given $a_{n}\left(m_{j}\right.$ dependent), the red curve is the Pareto frontier among which the modification will be selected.

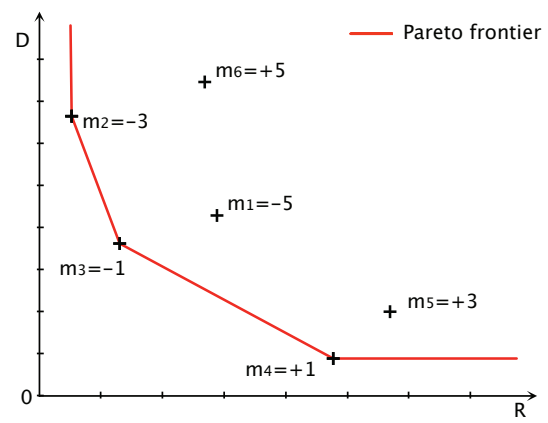

Fig. 1. Pareto frontier obtained for a given set of RD pairs.

2. Normalization of these pairs:

$$
\hat{R}_{n}^{w_{j}}=\frac{\left(\bar{R}_{n}^{w_{j}}-\bar{R}_{\text {min }}\right)}{\left(\bar{R}_{\text {max }}-\bar{R}_{\text {min }}\right)}, \hat{D}_{n}^{w_{j}}=\frac{\left(\bar{D}_{n}^{w_{j}}-\bar{D}_{\text {min }}\right)}{\left(\bar{D}_{\text {max }}-\bar{D}_{\text {min }}\right)}
$$

where $\left(\hat{R}_{n}^{w_{j}}, \hat{D}_{n}^{w_{j}}\right)$ is the normalized pair, $\left(\bar{R}_{\min }, \bar{R}_{\max }\right)$ and $\left(\bar{D}_{\min }, \bar{D}_{\max }\right)$ are the minimal and maximal rate and distortion among the Pareto-optimal pairs.
3. Selection of the best pair among these $\left(\hat{R}_{n}^{w_{j}}, \hat{D}_{n}^{w_{j}}\right)$ pairs by minimizing the euclidean $l^{2} \_$norm:

$$
\left(R_{\text {opt }}, D_{\text {opt }}\right)=\min \left\{\left\|\left(\hat{R}_{n}^{w_{j}}, \hat{D}_{n}^{w_{j}}\right)\right\|_{2}\right\}_{n \in[1, N], j \in[1,6]}
$$

Finally, the selected modification $\left(a_{n}, m_{j}\right)$ is the one associated to the optimal pair $\left(R_{o p t}, D_{o p t}\right)$. Note that the pairs with a too high distortion are rejected to preserve the prediction efficiency. The main advantage of this scheme compared to the classical RD-optimization is that it is independent of the Lagrangian multipliers assessment and then adaptive for each block. This approach allows to compare all the best pairs with a correct order of magnitude. However, the scheme does not take into account the quantization parameters, as a Lagrangian multiplier, function of QP, could.

Finally, the index extraction at the decoder side is expressed as $I_{i}=\left|S_{i}\right| \bmod 2$, where $I_{i}$ represents the extracted index of the $i^{\text {th }}$ block and $S_{i}$ is the sum of the decoded coefficients $a_{n}$. The MPM index is set to $I_{i}$ only if there is at least one non zero AC coefficient. Otherwise, the MPM index is traditionally read.

\section{EXPERIMENTAL RESULTS}

\subsection{Experimental settings}

The proposed scheme has been implemented into the JM KTA software [2] version 2.1. H.264/AVC reference results are generated using Intra $16 \times 16$ and Intra $8 \times 8$. The $8 \times 8$ DCT transform is enabled and CABAC entropy coding is selected.

The first experiment has been performed on eight 4:2:0 sequences, with resolutions from QWVGA to 1080p. Two ranges of Quantization Parameters (QP) have been selected to study the results from high to medium bitrates: QP set 1: 1217-22-27, QP set 2: 22-27-32-37. All bitrate savings are computed with the Bjontegaard metric [11] as recommended by VCEG. A test on several 4:4:4 sequences is also performed.

\subsection{Experimental results for YUV 4:2:0}

Table 1(a) gives the percentage bitrate saving for each sequence and two QP ranges. The scheme gives a systematic bitrate saving for all tested sequences and reaches $2.8 \%$ and $2.1 \%$ for sequences SunFlower and BlueSky 1080p. Unlike the previous scheme proposed for Inter-coding, the scheme efficiency is preserved at medium bitrate where the amount of coefficients is lower compared to high bitrate with respectively $1.5 \%$ and $1.6 \%$ bitrate saving in average. This is a significant gain relatively to the cost of the considered index on the Intra bitrate $(6 \%)$. When non-zero coefficients are available, i.e. the MPM index is not sent, Table1(b) gives the mark location. Coefficients are modified in $60 \%$ of the cases and indices are hidden mainly in the AC component at high bitrate whereas the DC plays a most important role at low bitrate. 


\begin{tabular}{|c|c|c|c|c|c|}
\hline QP range & Set 1 & Set 2 \\
\hline \hline Mobisode2 qwvga & 2.3 & 1.3 \\
\hline RaceHorses qwvga & 0.9 & 1.1 \\
\hline RaceHorses wvga & 1.0 & 1.0 \\
\hline DucksTakeOff 720p & 1.4 & 1.7 \\
\hline BlueSky 1080p & 1.3 & 2.1 \\
\hline Chroma DC & 19 & 27 & 31 \\
\hline DucksTakeOff 1080p & 1.6 & 1.8 \\
\hline Chroma AC & 38 & 33 & 29 \\
\hline SunFlower 1080p & 2.8 & 1.1 \\
\hline Tractor 1080p & 1.9 & 1.5 & 43 & 40 & 40 \\
\hline Average & $\mathbf{1 . 6}$ & $\mathbf{1 . 5}$ \\
\hline (a) & & & \\
\hline
\end{tabular}

Table 1. Percentage bitrate savings for the two set of QPs (a), and location of the mark (in percentage) for 3 QPs (b).

In addition to these objective results, a subjective assessment has been realized such as the one proposed in [3]. Similar conclusion is obtained: there is no visible degradation.

\subsection{Experimental results for YUV 4:4:4}

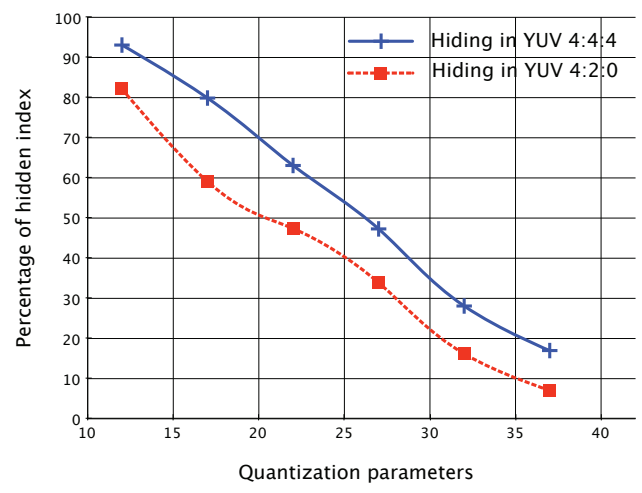

Fig. 2. Percentage of hidden index for the proposed scheme in YUV 4:2:0 and YUV 4:4:4 for each QPs.

We have tested the scheme on a set of 12 sequences available both in YUV 4:2:0 and YUV 4:4:4. Figure 2 gives the percentage of hidden index in average for the proposed scheme applied to the two formats, with QPs from 12 to 37 . The hiding in YUV 4:4:4 systematically allows to reduce the number of transmitted index compared to hiding in YUV 4:2:0 sequences. This reduction varies from $10 \%$ to $20 \%$ and it is larger for the QP 17 and 22 where the number of transform coefficients starts to drop down for YUV 4:2:0 sequences. For the scheme extended to YUV 4:4:4, the percentage of hidden index reaches $93 \%$ at high bitrate. As expected, less indices are transmitted for this color format. This is confirmed by an increase of the percentage bitrate savings which reach $2.60 \%$ for the sequence DucksTakeOff 720p for the second set of QPs in YUV 4:4:4. In average, the bitrate saving is increased by $36 \%$ and $47 \%$ in YUV 4:4:4 compared to YUV 4:2:0, for the first and the second set of QPs respectively.

\section{CONCLUSION}

An unconventional application of data hiding has been proposed in a previous work to perform video compression. MVComp indices were hidden in Chroma and Luma samples.

This paper further addresses this topic, and solves main remaining issues. The scheme is applied to the Intra Most Probable Mode. Dealing with Intra-coding allows to have a higher amount of non-zero transform coefficients available for hiding. As another new feature, a Pareto-based optimization is performed to drive the selection of the mark, and its location, avoiding the burden of a Lagrangian tuning. An average $1.5 \%$ (up to $2.8 \%$ ) bitrate saving is reported, which is a significant gain given that only a signalling index has been considered. The scheme has also been tested for $4: 4: 4$ color format sequences. As expected, an additional $47 \%$ increase of the previously reported bitrate saving is observed.

Applying data hiding techniques for video compression purposes raises several technical difficulties. This paper solves most of them, and shows that such a technique is of interest to improve the current standard. Especially, it is particularly adapted for reducing the cost of signalisation indices that tends to take more and more place in nowadays competition-based video codecs.

\section{REFERENCES}

[1] "Advanced Video Coding for Generic Audiovisual Services, ITU-T Recommendation H.264 and ISO/IEC 14496-10 (MPEG-4 AVC)," Standard Version 7: Apr. 2007, ITU-T and ISO/IEC JTC 1.

[2] K. Suehring, "H.264/AVC and KTA software coordination." Available: http://iphome.hhi.de/suehring/.

[3] J.-M. Thiesse, J. Jung and M. Antonini, "Data hiding of Motion Information in Chroma and Luma Samples for Video Compression," MMSP'10, France, Oct. 2010.

[4] F. Hartung and B. Girod, "Watermarking of Uncompressed and Compressed Video", in Sig. Proc., 66(3):283-301,1998.

[5] O. Nemethova, G. Calvar Forte, and M.Rupp, "Robust Error Detection for H.264/AVC Using Relation Based Fragile Watermarking," IWSSIP, Budapest, Hungary, Sep. 2006.

[6] L. M. Marvel, G. W. Hartwig, C. Boncelet, "Compression Compatible Fragile and Semi-Fragile Tamper Detection," SPIE Int. Conf. on Security and Watermarking of Multimedia Contents II, vol. 3971, USA, 2000.

[7] P. Campisi, D. Kundur, D. Hatzinakos, and A. Neri, "Compressive Data Hiding: An Unconventional Approach for Improved Color Image Coding," EURASIP JASP, 152-163, 2002.

[8] M. Chaumont, and W. Puech, "A DCT-Based Data-Hiding Method to Embed the Color Information in a JPEG Grey Level Image," EUSIPCO, Italy, Sep. 2006.

[9] B. Zhu, and A. H. Tewfik, "Media Compression via Data Hiding," $31^{\text {st }}$ Asilomar Conf. on SSC, vol. 1, pp. 647-650, 1997.

[10] G. Laroche, J. Jung, and B. Pesquet-Popescu, "RD optimized coding for motion vector predictor selection," IEEE Trans. on CSVT, 18(9) :1247-1257, Sep. 2008.

[11] J. Jung and S. Pateux, "An Excel add-in for computing Bjontegaard metric and its evolution," ITU-T VCEG contribution VCEG-AE07, Marrakech, Jan. 2007. 